\section{Isolated substantia nigra involvement in brainstem demyelination}

Sir,

Focal signal changes in substantia nigra only have been reported in St. Louis encephalitis. ${ }^{[1]}$ The involvement is more diffuse apart from substantia nigra with areas of hemorrhage, infarction, and edema in Eastern equine encephalitis, West Nile viral encephalitis, Japanese encephalitis, and tick-borne encephalitis. ${ }^{[2,3]}$ Involvement of deep grey nuclei in acute disseminated encephalomyelitis (ADEM) has been described. There are no reports of isolated symmetrical substantia nigra involvement in brainstem demyelination that have been reported so far. Here, we report a lady with acute onset right crural monoplegia with neuroimaging showing bilateral symmetrical hyperintensity in substantia nigra only. She had good response to steroids suggesting the lesion to be demyelination.

A 45-year-old lady presented with weakness of right lower limb of 1 day duration. The weakness was sudden in onset, nonprogressive. There was no speech disturbance or upper limb weakness. No sensory symptoms, bladder or bowel disturbances. No complaints of fever, seizures or headache. There was no history of travel outside the country. She had upper respiratory tract infection three weeks before the present symptoms. On examination, she was conscious and oriented, speech and cranial nerves were normal. She had right lower limb weakness of medical research council (MRC) grade $2 / 5$ with sluggish right knee and ankle jerks. No sensory loss. Plantar response was extensor on the right side. No meningeal signs. Complete hemogram renal, hepatic and thyroid function tests were normal. Brain magnetic resonance imaging (MRI) showed symmetrical T2/fluid-attenuated inversion recovery (FLAIR) hyperintensities in bilateral substantia nigra without any enhancement on contrast [Figure 1]. MRI of the spinal cord did not reveal any intramedullary signal abnormality. Cerebrospinal fluid (CSF) examination showed normal cell count of 6 cells, glucose of $64 \mathrm{mg} / \mathrm{dl}$ with elevated protein $(76 \mathrm{mg} / \mathrm{dl})$. Oligoclonal bands (OCB) were weakly positive. CSF polymerase chain reaction (PCR) for herpes simplex virus, Dengue virus serotype 1-4, West Nile virus, Japanese encephalitis, Nipah virus, Coxsackie virus, Echovirus, Enterovirus 70-72, Cytomegalovirus,
Human Herpes virus-6 were negative. CSF-tubercular bacilli (TB) PCR was negative. Serological testing for antinuclear antibody (ANA) and antineuromyelitis optica (anti-NMO) antibody was negative. Serological testing for human immunodeficiency virus (HIV) and hepatitis B virus (HBsAg) was negative. The chest $\mathrm{X}$-ray and ultrasonography of the abdomen and pelvis were normal. Based on the clinical picture, imaging findings, CSF-OCB positivity and after ruling out the prevalent etiologies of viral encephalitides, a diagnosis of brainstem demyelination was made. She was given injection methylprednisolone $1 \mathrm{~g}$ daily for 5 days. There was gradual improvement in lower limb weakness and was able to ambulate with support. She was put on tapering dose of oral prednisolone. Repeat brain MRI after 14 days showed decrease in the lesion [Figure 1].

Postinfectious encephalomyelitis typically involves the white matter; however, lesions in grey matter have also been seen. Basal ganglia, thalamus, brainstem and even cortical grey matter may be involved in postinfectious encephalomyelitis. ${ }^{[4]}$ The clinical and imaging findings of our patient pointed toward brainstem demyelination as a possibility since all other possible etiologies for viral encephalitis in this part of world were ruled out. Isolated symmetrical substantia nigra involvement in brainstem demyelination has not been reported. Involvement of substantia nigra has been reported in viral encephalitis. In Eastern equine encephalitis, West Nile viral encephalitis, Japanese encephalitis, and tick-borne encephalitis, the involvement is more diffuse apart from substantia nigra with areas of hemorrhage, infarction, and edema. ${ }^{[2,3]}$ Involvement of substantia nigra in such an isolated or selective fashion has not been reported in these viral encephalitides. Isolated signal changes in substantia nigra have been reported in St. Louis encephalitis only. ${ }^{[1]}$ An exhaustive review of the literature for other similar distribution of focal signal changes of the substantia nigra in any brain disease that included infectious, metabolic, and degenerative disorders was done. Isolated substantia nigra involvement has been described in encephalitis lethargica wherein the patient presented with clinical features suggestive of parkinsonism. ${ }^{[5]}$ St. Louis encephalitis is not prevalent in this part of the world. Our patient did not present with fever, headache or altered level of consciousness to consider possibility of viral encephalitis.

This is the first report in the literature on the atypical imaging findings in brainstem demyelination in the form of isolated, focal signal changes in substantia nigra without contrast enhancement. There was response to steroids. 

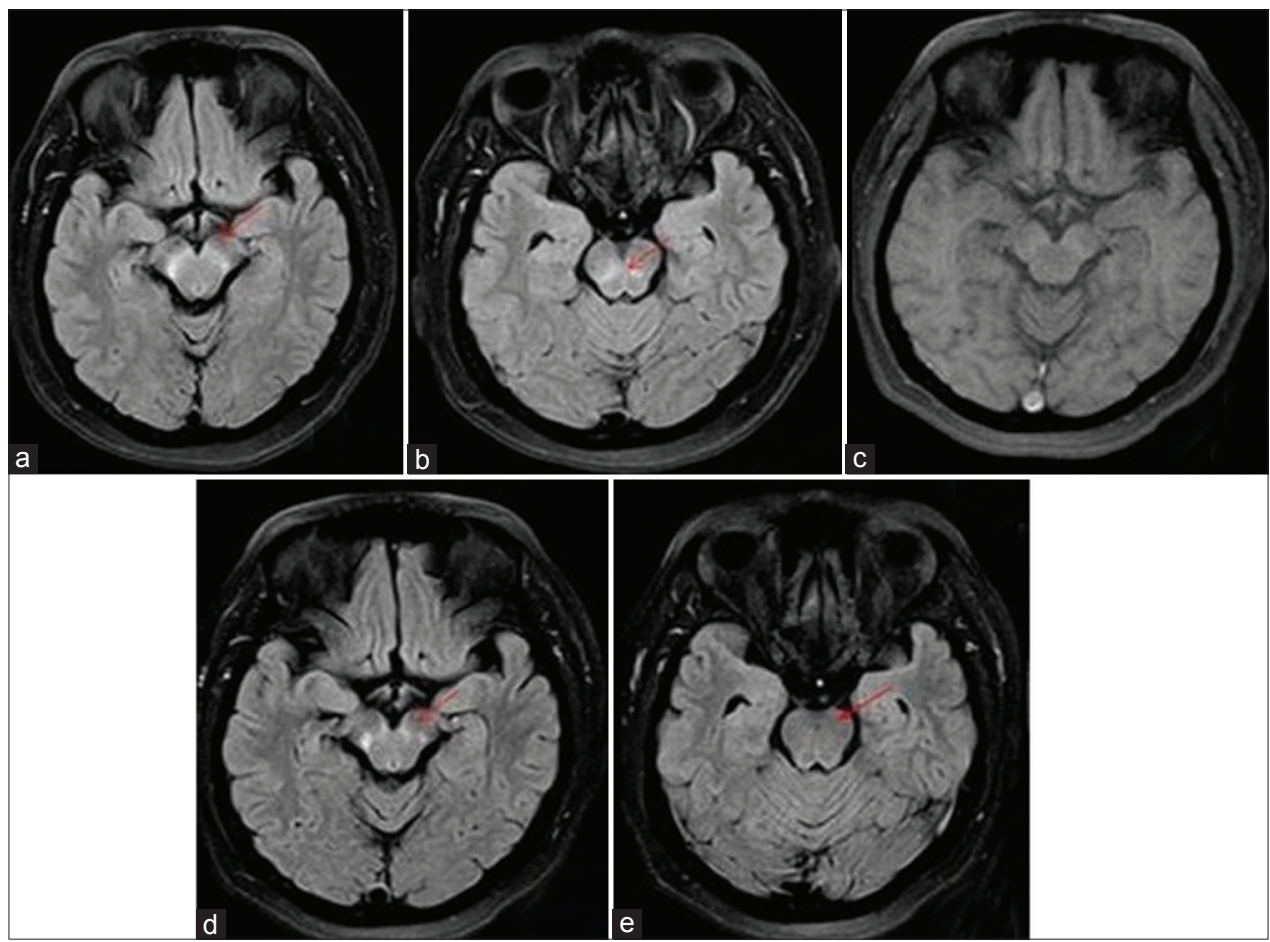

Figure 1: (a and b) MRI brain FLAIR images axial view showing symmetrical hyperintensity in bilateral substantia nigra (red arrow). (c) Post contrast T1 MR images showing no contrast enhancement. ( $d$ and e) MRI brain FLAIR images axial view showing decrease in size of the hyperintensities (red arrow)

Rohan R. Mahale, Anish Mehta, Srinivasa Rangasetty

Department of Neurology, MS Ramaiah Medical College and Hospital, Bangalore, Karnataka, India

\section{Address for correspondence:} Dr. Rohan R. Mahale, Department of Neurology, MS Ramaiah Medical College and Hospital, Bangalore - 560 054, Karnataka, India.

E-mail: rohanmahale83@gmail.com

\section{References}

1. Cerna F, Mehrad B, Luby JP, Burns D, Fleckenstein JL. St. Louis encephalitis and the substantia nigra: MR imaging evaluation. AJNR Am J Neuroradiol 1999;20:1281-3.

2. Pradhan S, Pandey N, Shashank S, Gupta RK, Mathur A. Parkinsonism due to predominant involvement of substantia nigra in Japanese encephalitis. Neurology 1999;53:1781-6.
3. Shoji H, Kida H, Hino H, Matsuura S, Kojima K, Abe T, et al. Magnetic resonance imaging findings in Japanese encephalitis. White matter lesions. J Neuroimaging 1994;4:206-11.

4. Garg RK. Acute disseminated encephalomyelitis. Postgrad Med J 2003;79:11-7.

5. Kun LN, Yian SY, Haur LS, Tjia H. Bilateral substantia nigra changes on MRI in a patient with encephalitis lethargica. Neurology 1999;53:1860-2.

\begin{tabular}{|l|l|}
\hline \multicolumn{2}{|c|}{ Access this article online } \\
\hline Quick Response Code: & Website: \\
\hline & www.ruralneuropractice.com \\
\hline & \\
\hline
\end{tabular}

